\title{
A Coarse-Grained Model for Free and Template-Bound Porphyrin Nanorings
}

\author{
David Ormrod Morley, ${ }^{\dagger}$ Marc Malfois, ${ }^{\ddagger}$ Nuntaporn Kamonsutthipaijit, ${ }^{\S}$ Dmitry V. Kondratuk, ${ }^{\ddagger}$ \\ Harry L. Anderson, ${ }^{\S}$ and Mark Wilson ${ }^{* \dagger} \dagger$ \\ ${ }^{\dagger}$ Department of Chemistry, Physical and Theoretical Chemistry Laboratory, University of Oxford, South Parks Road, Oxford OX1 \\ 3QZ, U.K. \\ ${ }^{\ddagger}$ Diamond Light Source Ltd, Harwell Science and Innovation Campus, Didcot OX11 0DE, U.K. \\ ${ }^{\S}$ Department of Chemistry, University of Oxford, Chemistry Research Laboratory, Mansfield Road, Oxford OX1 3TA, U.K.
}

\section{Supporting Information}

ABSTRACT: Coarse-grained simulation models are developed to study both template-bound and free porphyrin nanoring systems. Key interactions are modeled with relatively simple (and physically motivated) energy functions which allow for relatively facile transfer both between different ring
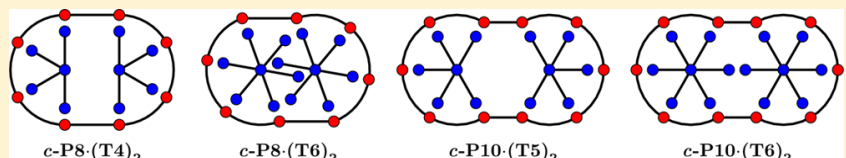

sizes and between the template-bound and free nanoring systems. The effects of varying the model parameters on the respective radii of gyration are determined. The effects of including different templates on the ring structure are investigated both in terms of the detailed geometry of the template and the interaction strength between the template and the metal centers in the nanorings. The role of the template-nanoring interaction strength in controlling potential "caterpillar track" rotational motion is discussed. The relationship of the model to experimental small-angle X-ray, exchange spectroscopy, and electron spin resonance results is discussed.

\section{INTRODUCTION}

The study of the chemistry of porphyrin systems has been an area of intense investigation and rapid progress over the past century. The initial interest in these systems arose as the role of porphyrin rings as building blocks in biological macromolecules (e.g., hemoglobin and chlorophyll) was identified. The basic structure of the porphyrin ring as a framework of four pyrroles connected by methene linkages was proposed by Küster ${ }^{1,2}$ in 1912 and not long after when the first Nobel Prize in the field was awarded to Fischer in 1930 for the synthesis of hemein, an iron-containing porphyrin. The focus of this study originates from the resolution of the crystal structure of $\mathrm{LH} 2$, a light harvesting complex in photosynthetic bacteria, which was shown to contain ring-like arrays of porphyrin units. Synthetic pathways to these cyclic materials utilize a template-directed approach used initially to form a ring containing eight butadiyne linked porphyrin units (termed $\boldsymbol{c}$ P8). ${ }^{56}$ Following this success, systematic modifications to the templates has led to the synthesis of a wide range of nanorings, with rings in the range $\boldsymbol{c}$-P5 to $\boldsymbol{c}$-P50 becoming available. ${ }^{7-11}$

The porphyrin systems have been investigated by a number of experimental methods with photoluminescence (PL) spectroscopy confirming their ultrafast energy transfer and light harvesting capabilities, ${ }^{12-14}$ and making them excellent prototype materials for use in next generation solar cells. ${ }^{15,16}$ Scanning tunnelling microscopy (STM) has shown that nanorings exhibit stacking behavior when deposited on an $\mathrm{Au}(111)$ surface using electrospray techniques, with the formed nanopore arrays able to trap and organize adsorbed molecules such as $\mathrm{C}_{60} \cdot{ }^{17,18}$ In addition small-angle $\mathrm{X}$-ray scattering (SAXS) has been used to explore the structure and flexibility of the nanorings in solution. ${ }^{19,20}$ The structural characterization of porphyrin nanorings has been achieved primarily through a combination of ${ }^{1} \mathrm{H}$ NMR, X-ray crystallography, and SAXS. Of these techniques, SAXS can most readily provide information regarding the conformations adopted in solution. SAXS data have been published for the free rings $\boldsymbol{c}$-P6 and $\boldsymbol{c}-\mathbf{P} 12^{8,19,20}$ and template-bound $c$-P8.(T4) ${ }_{2}$ and $c$-P10.(T5 $)_{2}{ }^{21}$ although data have been collected for a number of additional ring sizes. ${ }^{10}$

Recently, attention has turned to the study of the templatebound nanorings. These may be generated either as a direct product of synthesis, where the template acts as a scaffold for the coupling of porphyrin subunits, ${ }^{8,9,19,21}$ or via the addition of an excess of template molecules to a solution of the free nanoring. ${ }^{22}$ Although the templates were intended as scaffolding for use in the synthesis process, their complexes with nanorings have been found to have noteworthy structural and dynamical properties in their own right, in particular in promoting the stabilization of novel ring conformations. The considerable interest in these structures arises from the fact that the templates introduce rigidity into the otherwise flexible nanorings, leading to fully conjugated cyclic porphyrin frameworks which may be shape-persistent in solution. These molecules possess potentially unique electronic and dynamical

Received: May 31, 2017

Revised: July 12, 2017

Published: July 13, 2017 
properties, which have been probed by $\mathrm{PL},{ }^{13,14} \mathrm{EPR},{ }^{22}$ and exchange spectroscopy, EXSY. ${ }^{21}$

In recent years there have been huge advances in investigating synthetic systems which display controlled motion on the molecular level, including motors, rotors, gears, ratchets, ball-bearings, and switches; with the overall aim of developing molecular machines. ${ }^{23}$ Some template-bound porphyrin nanorings have been shown to exhibit correlated molecular motion. ${ }^{21}$ For example, the template-bound porphyrin systems $c$-P8. $(\mathrm{T} 4)_{2}, c$-P8.(T6) ${ }_{2}, c$-P10.(T5) ${ }_{2}$, and $c$-P10.(T6) ${ }_{2}$ have been investigated by EXSY. These 1:2 nanoring-to-template ensembles are known as "caterpillar track" (CT) complexes. On proton irradiation $\boldsymbol{c}$-P8.(T6) ${ }_{2}$ showed exchange signals only between environments related by $60^{\circ}$ conrotatory motion of the template molecules. A similar result was found for $\boldsymbol{c}$-P10. (T6) $)_{2}$ but, over time, signals corresponding to first $120^{\circ}$ then $180^{\circ}$ rotations build up-a characteristic sign for a stepwise rotation mechanism. However, analogous experiments on complexes without free binding sites on the template molecules $(c \text {-P8.(T4 })_{2}, c$-P10.(T5 $\left.)_{2}\right)$ showed no exchange signals. It appears, therefore, that $\mathrm{CT}$ complexes which have free template binding sites undergo correlated motion with both templates rotating coherently.

Alternatively, the conformation may be controlled through variation of the metal occupying the porphyrin subunits, as a result of the different binding affinities to the template molecules. Including paramagnetic species such as $\mathrm{Cu}$ allows these systems to be examined by electron paramagnetic resonance (EPR). ${ }^{22}$ Dimetallic porphyrin nanorings were originally synthesized with the aim of developing another method of probing the solution-phase structure. By replacing a number of zinc centers with copper, it is possible to measure distances within the oligomers by EPR. The different binding affinities of the metals to the template allow the nanoring conformation to be altered by appropriate selection of the template geometry. ${ }^{22}$ In addition, the self-assembly of nested structures (such as the so-called "Russian Doll" complexes in which a small ring sits inside a larger conformation) have been reported using a mixture of separate aluminum and zinc species. $^{24}$

The motivation for studying complex porphyrin frameworks is clear, with the prospect of developing self-assembling conformationally controllable supramolecular structures which may display ultrafast exciton dynamics. However, developing reliable synthetic pathways is complex and time-consuming. As a result, computational studies are essential to complement ongoing experimental investigations. Electronic structure calculations offer potentially the greatest detail of information including, crucially, that required to consider directly the electron dynamics. However, the computational expense for studying systems of the size required is high. The development of simpler, potential model-based, methods will allow for a systematic study of the factors controlling the ring structure (including the role of the templates) while retaining the possibility of feeding into higher level electronic structure calculations. Our aim is to develop a "bottom-up" computational model based on relatively simple potentials which allow the capacity to explore both the structure and dynamics of a wide range of porphyrin systems. A previous attempt to model porphyrin rings using a Monte Carlo method ${ }^{17}$ employed a bond angle constraint by limiting the bending angle between metal atoms using hard discs.
Our approach is to construct a coarse-grained model in which relatively complex molecular subunits are replaced with single interaction sites. The aims are to aid the understanding of key experimental observations, as well as developing models which transfer both between different ring sizes and between template-bound and free nanoring systems via a physically motivated systematic variation in the model parameters.

We will first explore the structure of free porphyrin nanorings (without templates). This is of interest because while the crystalline structure has been well characterized through X-ray crystallography (see, for example, ref 25), the solution phase has proven much more difficult to probe. Despite SAXS techniques continually improving, it is still notoriously difficult to gather good quality scattering data on the smaller length scales found in these nanorings. ${ }^{26}$ Simulation models allow the scattering functions and distance distribution functions to be calculated directly from the atom coordinates and compared to experimental results. To highlight the potential flexibility of the proposed approach, template molecules will be introduced into the nanoring structures, and the effects on the flexibility and geometry of the nanorings will be investigated. In addition we will study the dynamics of $c$ P10.(T6) $)_{2}$ directly observing a mechanism for the rotational molecular motion. Finally, the model will be extended to include metalloporphyrin units in the template-bound nanoring frameworks which have different metal-template binding affinities.

\section{EXPERIMENTAL SETUP}

Synchrotron radiation SAXS data were collected using standard procedures on the $\mathrm{I} 22$ beamline equipped with a photon counting detector. The beam was focused onto the detector placed at a distance of $1.25 \mathrm{~m}$ from the sample cell. The covered range of momentum transfer was $0.03<q<1.0 \AA^{-1}(q$ $=4 \pi \sin (\theta) / \lambda$ where $2 \theta$ is the scattering angle and $\lambda=1.00 \AA$ is the X-ray wavelength). SAXS measurements were performed in toluene/1\% pyridine at known concentrations $\left(\sim 10^{-4} \mathrm{M}\right)$ in a cell with mica windows ( $1 \mathrm{~mm}$ path length).

\section{MODELING THE PORPHYRIN SYSTEMS}

III.A. Overview. The basic porphyrin building blocks consist of metalloporphyrin rings joined via butadiyne linkages, as shown in Figure 1a. The porphyrin ring consists of four pyrroles connected together to form a scaffold in which a metal atom can sit in square-planar coordination with the pyrrole nitrogens. Because of the "scaffolding" in the porphyrin unit, the ring itself is largely inflexible and it is the butadiyne linkages in which most of the strain is localized. ${ }^{19}$ The challenge when modeling these porphyrin systems is to accurately replicate the physics of the system using a coarse-grained model. The choice of model must reproduce the intermetal distance and angle distributions by correctly capturing the flexibility of the oligomer. This is a nontrivial problem as the distortion of the butadiyne linkages can be quite significant. For example, the crystal structure of $\boldsymbol{c}$-P6.(T6) shows a metal-metal distance of $\sim 12.2 \AA$ compared to the $\sim 13.5 \AA$ observed for the linear dimer (Figure $1 \mathrm{a}^{19}$ ). Developing a coarse-grained model can, of course, be achieved in any number of different ways. Our primary source of structural information here is SAXS, for which the signal would be expected to be dominated by scattering from the metal centers. As a result, the metal centers are retained and a framework set up around them. The 


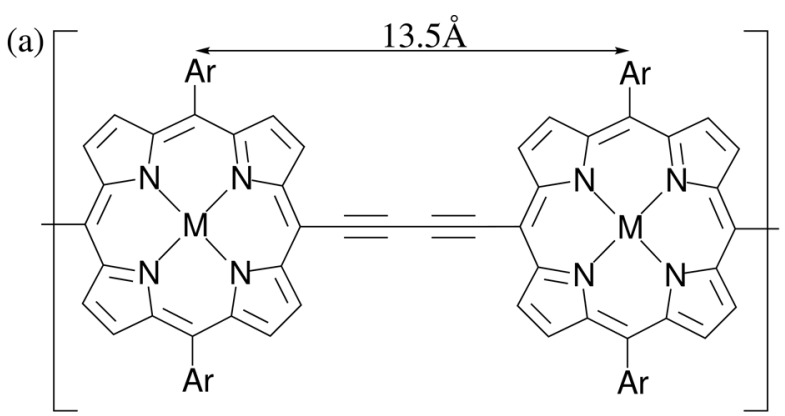

(b)
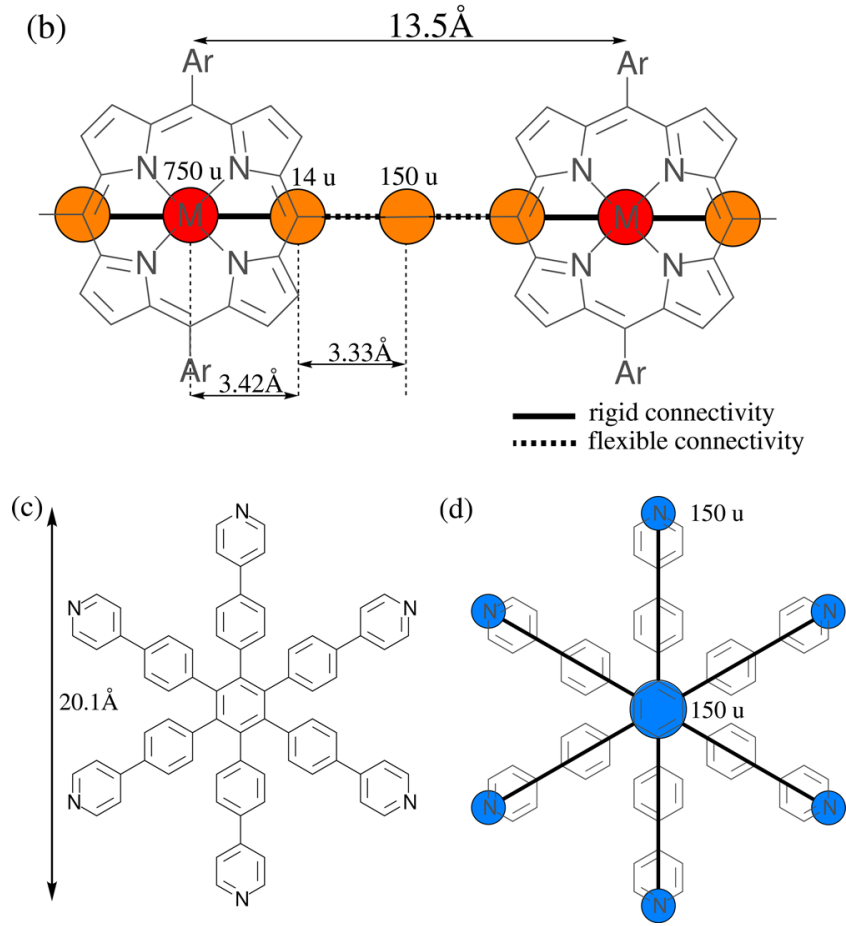

Figure 1. (a) The chemical structure of the basic porphyrin subunit which makes up the nanorings showing the butadiyne-linked metalloporphyrin rings. (b) Schematic diagram of the coarse-grained centers used to model the porphyrin subunits in the simulation models. The large circles indicate the coarse-grained interaction centers along with their associated masses. The full and dashed lines indicate the use of rigid and flexible intercenter interactions, respectively. (c) An example of a typical template molecule, T6. (d) Schematic of the coarse-grained centers species used to model T6 in the simulation model. The circles again represent the coarse-grained centers.

porphyrin units themselves are expected to be relatively rigid (see, for example, the discussion in ref 19) and so can be effectively coarse-grained without significant loss of confomational information. Molecular dynamics is used throughout as a key target to attempt to rationalize the potentially complex coherent motion associated with, for example, the so-called "caterpillar track" motion. ${ }^{21}$

III.B. Free Nanorings (no Templates). Figure $1 \mathrm{~b}$ shows the basic coarse-grained model employed containing the following contributions:

1. The porphyrin ring is reduced to a three site basis. The key component of the ring is the metal position which dominates many experimental observables, and hence is required for the calculation of properties such as the pair distribution functions, structure factors, or radii of gyration. As a result the metal center is retained and the mass of the porphyrin unit is concentrated at this point.

2. The central metal atom is connected to "placeholder" atoms on either side of a fixed relative position, which define the extent of the rigid porphyrin section.

3. The butadiyne linkage is replaced by a single atom placed midway between the porphyrin rings. It is joined to the two surrounding placeholder atoms by rigid bonds of given length. However, unlike the metal-placeholder connections, there are additional degrees of freedom available so that it may move in space relative to its neighbors, provided that the length of the rigid connections are maintained.

4. A further physical consideration is how to incorporate angle strain effectively into the model. Angle strain is the energetic cost arising from the distortion of bond angles from their ideal values. Previous work on these ring systems introduced the bond angle constraint by placing hard discs at the midpoints between the metals, the overlapping of which incurred an infinite energy cost. ${ }^{17} \mathrm{~A}$ shifted truncated repulsive $24-12$ potential $(24-12 \mathrm{P})$ is set up between all next-nearest metal neighbors in the oligomer, with $r_{\min }=27.0 \AA$, corresponding to the distance between these two metals with a M-M-M bond angle of $180^{\circ}$. The result is that a triplet of metal atoms will experience no repulsive potential only when adopting a linear configuration. As a bending angle is introduced the next-nearest metal distance will decrease and there will be a repulsive force acting between the two end atoms. This potential can be tuned by varying the parameter $\epsilon$ in the 24-12P (which will be termed $\epsilon_{\text {ang }}$ ). Increasing the value of $\epsilon_{\text {ang }}$ will increase the restoring force for the same deviation resulting in a reduction in the range of accessible angles.

5. The terms described above work well replicating bond angle distributions but do not account for changes in the intermetal distance for nearest neighbors as a consequence of any distortion of the butadiyne linkages. Given the size of the distortion it is very important that this effect be incorporated into the model. Again a 24$12 \mathrm{P}$ is used which acts between nearest metal neighbors with $r_{\text {min }}=13.5 \AA$ A . The atom placed centrally between them can now act as a hinge point, so that as it moves away from the direct metal-metal vector the intermetal distance must shorten to maintain the length of the rigid connections. The extent to which the backbone is allowed to pucker in this way is controlled through the parameter analogous to $\epsilon$ in the 24-12P (here termed $\left.\epsilon_{\text {dist }}\right)$. Increasing $\epsilon_{\text {dist }}$ acts to stiffen the hinge and reduce the distortion.

III.C. Template-Bound Molecules. Many of the porphyrin frameworks of interest still contain one or more template molecules which were used as "scaffolding" for the construction of the oligomer. These templates consist of rigid hydrocarbon "spokes" terminating with a pyridine functional group. A typical example is shown in Figure 1c. The nitrogen of the pyridine unit may bond to the metals in the porphyrin rings by donating its lone pair into vacant orbitals on the metals. The strength of the interaction is typically $<100 \mathrm{~kJ} \mathrm{~mol}^{-1}$, relatively weak compared to covalent bonding, and is dependent on the identity of the metal centered in the porphyrin unit. For 
Table 1. Radii of Gyration for Free Porphyrin Nanorings (No Templates) As Derived from Experimental SAXS Data, in Å

\begin{tabular}{lcccccccc} 
& $c-P 6$ & $c-P 7$ & $c$-P8 & $c$-P10 & c-P12 & $c$-P16 & c-P18 & 29.93 \\
$R_{\mathrm{g}}$ (lower) & 10.86 & 13.16 & 16.14 & 18.91 & 23.34 & 37.96 \\
$R_{\mathrm{g}}$ (upper) & 15.81 & 15.70 & 19.53 & 22.37 & 25.51 & 32.80 \\
$R_{\mathrm{g}}$ (mean) & 12.97 & 14.76 & 17.83 & 21.20 & 25.36 & 31.42 \\
\hline
\end{tabular}
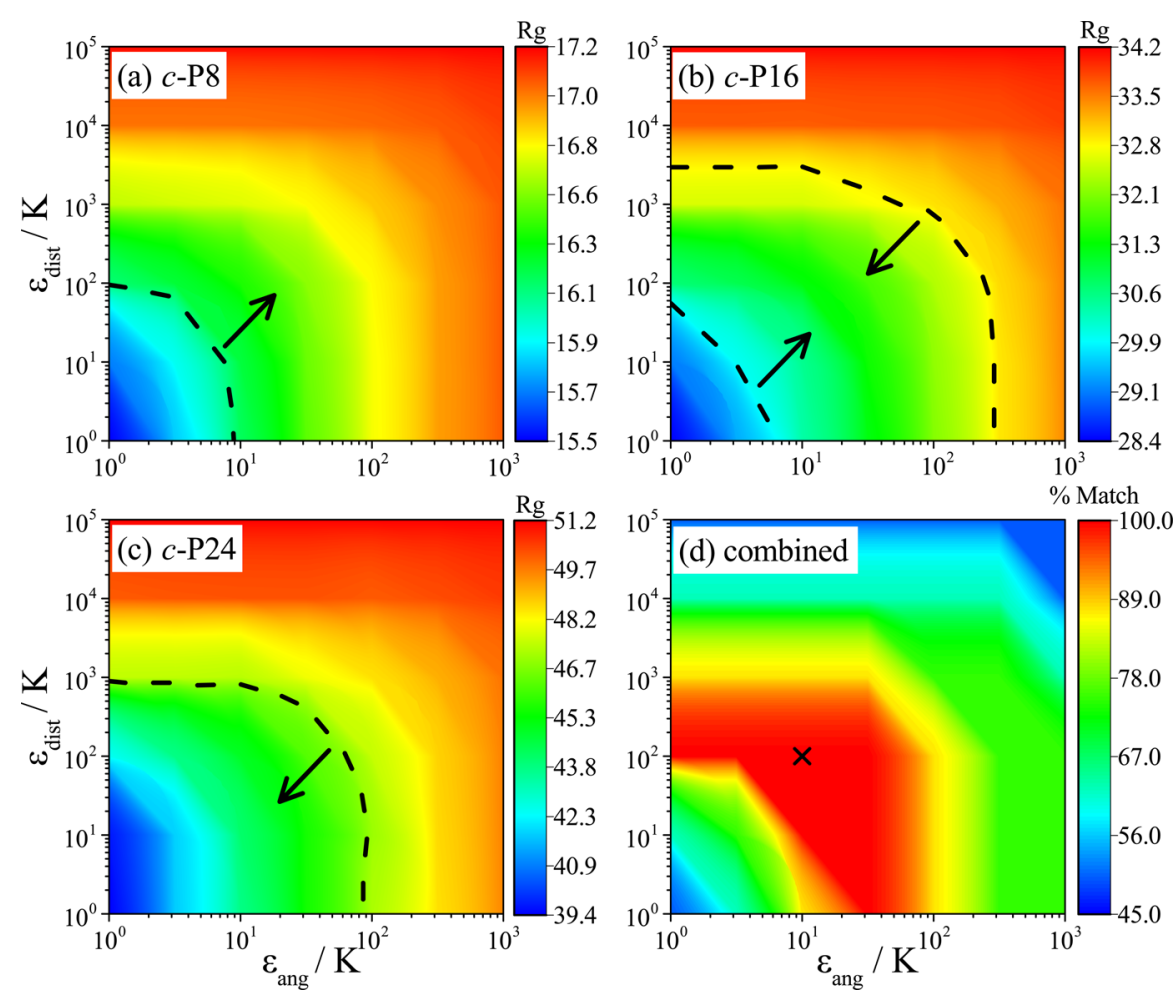

Figure 2. Searches over the parameter space $\left\{\epsilon_{\text {ang, }} \epsilon_{\text {dist }}\right\}$ for selected free nanorings (no templates), (a) c-P8, (b) c-P16, (c) c-P24. Panel d shows the results averaged over all the rings studied. In all panels the black lines indicate acceptable bounds according to experimental data. Panel $\mathrm{d}$ highlights the combinations of the parameters $\epsilon_{\text {ang }}$ and $\epsilon_{\text {dist }}$ which lead to agreement of the simulated values and agreement with experimental radii of gyration for all of the nanorings studied in the range $c$-P6 to c-P24.

example, the axial coordination of $\mathrm{Zn}$ has been estimated at $\sim 19 \mathrm{~kJ} \mathrm{~mol}^{-1}$ and the Cu-pyridine interaction at $\sim 6.2 \mathrm{~kJ} \mathrm{~mol}^{-1}$ from a consideration of differences in formation free energies. $^{27,28}$ In our model the template is again described with a reduced atomic basis. Atoms are placed at the positions of the nitrogens in the true template and rigidly connected to a central node. The mass of each spoke is concentrated at the position of the nitrogen as shown in Figure 1d.

The bonding between the nitrogen atoms in the template and the metal atoms in the porphyrin units is described by a Morse potential ${ }^{29}$ as it can explicitly account for bond breaking effects which will allow the template to dissociate from the porphyrin oligomer, potentially facilitating template mobility. This form gives excellent agreement with the potential calculated from Density Functional Theory (DFT; see below). The Morse potential has the form

$$
V(r)=D_{\mathrm{e}}\left[1-\mathrm{e}^{-\alpha\left(r-r_{0}\right)}\right]^{2}
$$

where $D_{\mathrm{e}}$ determines the energy well depth (and so controls the dissociation energy), $\alpha$ controls the width of the energy well, and $r_{0}$ defines the distance for the energy minimum. These parameters can be specified for every nitrogen-metal pair in the system, so that the potential may be tuned to the nature of the metal and enabling multimetal systems to be investigated.

\section{PARAMETRIZING PORPHYRIN SYSTEMS}

Two key parameters, $\epsilon_{\text {ang }}$ and $\epsilon_{\text {dist }}$ control the flexibility of the oligomer and require calibration. It is worth emphasizing that a central aim here is to assess whether a single parameter set can be effectively employed to model a chemically related set of systems (here meaning related in terms of rings constructed from the same basic building blocks). If successful then such a model could be applied in a predictive sense to study systems not yet fully synthesized. A useful procedure, which will help rationalize the respective values of the different parameters, is to search over a significant range of parameter space and compare the results to experimental observations. The experimental data used is the radius of gyration, $R_{\mathrm{g}}$, of free porphyrin nanorings as determined from the SAXS data presented here. ${ }^{10}$ The SAXS data provide reliable estimates for $R_{\mathrm{g}}$ from the fit to the low $k$ regime. In addition, a good range of free nanoring sizes have been studied and, as a result, $R_{\mathrm{g}}$ might be expected to vary in a systematic manner. This is commensurate with our ideal of building models transferable both in terms of ring size and template identity.

To determine the optimum combination of the simulation system size and time-scale, a series of simulations were carried out on $\boldsymbol{c}$-P24 ensembles with $\boldsymbol{c}$-P24 chosen as it is the most flexible of the rings investigated and hence would be expected to show the largest fluctuations in $R_{\mathrm{g}}$. Simulations were 

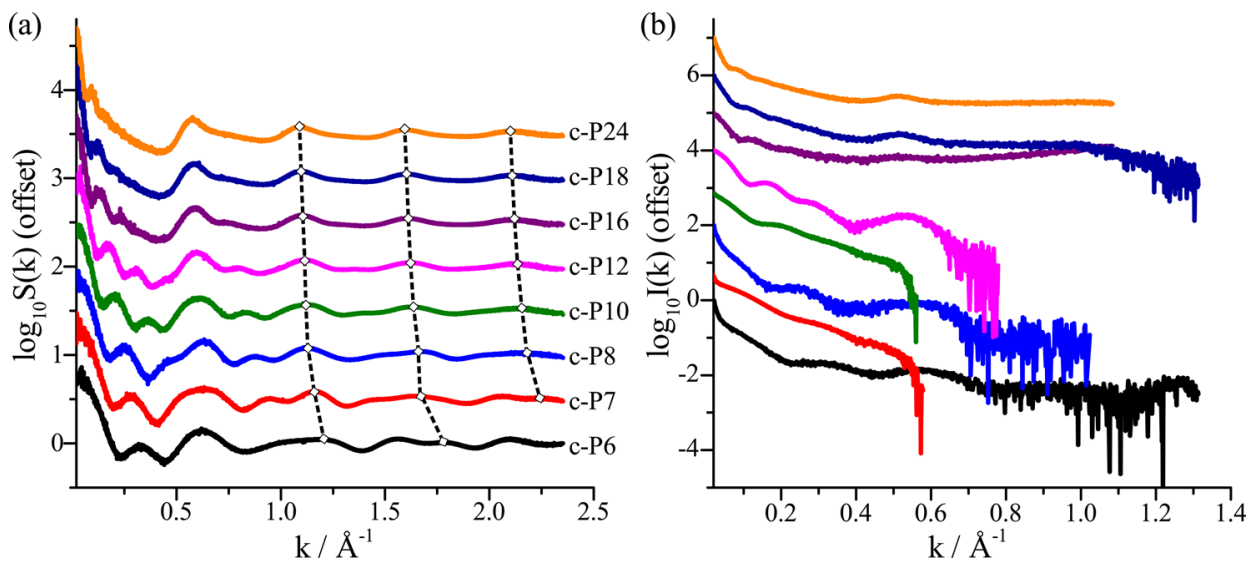

Figure 3. Comparison of the total scattering functions obtained from (a) simulation and (b) SAXS experiment. ${ }^{10}$ In both panels successive functions are offset along the ordinate axis for clarity. The colors of the lines in panel $b$ match those in panel a. In panel a the vertical lines highlight trends in peak positions as the ring size changes.

performed for varying system size over $\sim 1.5$ ns. Figure S1a shows the evolution of the fluctuations of the radii of gyration for eight porphyrin rings as indicated. The respective gyration radii are determined directly from the particle positions with the figure showing the percentage fluctuations from the mean value for each ring. As a result, the systems simulated contain 20 rings using a total simulation time of around $1 \mathrm{~ns}$ with the first 500 ps used for equilibration.

Experimental values for the respective radii of gyration were obtained by performing Guinier analysis on SAXS data for porphyrin nanorings. ${ }^{10}$ The effective extraction of $R_{\mathrm{g}}$ from the SAXS data requires careful treatment. ${ }^{30}$ Aggregation of particles may lead to distortions of $S(k)$ at low $k$ (i.e., in the region of interest) affecting the range of $k$-space in which the Guinier approximation is valid. Ideally the condition on the maximum extent of $k$ is that $k_{\max } \cdot R_{\mathrm{g}}<1.0$ but this condition is often relaxed so that $k_{\max } \cdot R_{\mathrm{g}}<1.3$ to allow a sufficient range of $k$ values to contribute to the calculation. ${ }^{31}$ As a result a procedure was developed to extract sensible upper and lower bounds for the radii of gyration. An example of the upper and lower bounds found by this method for $\boldsymbol{c}$-P12 is shown in Figure S1b with results for all ring sizes listed in Table 1.

To investigate the effect of varying key parameters, $\epsilon_{\mathrm{ang}}$ and $\epsilon_{\text {dist }}$ they were varied systematically between $\epsilon_{\text {ang }} / k_{\mathrm{B}}$ and $\epsilon_{\text {dist }} / k_{\mathrm{B}}$ of $10^{\circ} \rightarrow 10^{3} \mathrm{~K}$ and $10^{\circ} \rightarrow 10^{5} \mathrm{~K}$, respectively. The results for $c$-P8, c-P16, and $c$-P24 are summarized in Figure 2 panels a-c, where the values deemed acceptable according to the SAXS data have been highlighted (indicated as the "upper" and "lower" values in Table 1). The figure shows that these variables give the required fine control over $R_{\mathrm{g}}$. The nanoring may be stiffened by either increasing the angle strain or tightening the hinge which accounts for distortions in the butadiyne backbone, resulting in an increase in $R_{\mathrm{g}}$. It also demonstrates the additional flexibility which accompanies the larger nanoring systems due to their increased number of degrees of freedom. For $\mathbf{c}$-P6 we see an increase in $R_{\mathrm{g}}$ of $7.5 \%$ over the tested parameter space, whereas for $\mathbf{c}$-P24 the analogous value is $30.0 \%$. The consequence for parametrization is that these larger rings become more significant, as the values selected for $\epsilon_{\text {ang }}$ and $\epsilon_{\text {dist }}$ will have a greater effect on their structure. Figure $2 \mathrm{~d}$ shows the information from each individual ring merged to find the optimum values of $\epsilon_{\text {ang }}$ and $\epsilon_{\text {dist }}$. As can be seen there is a clear region which best fits with the experimentally derived values. From this we can conclude that the flexibility of the porphyrin subunits can be most accurately modeled by using values of $\epsilon_{\mathrm{ang}} / k_{\mathrm{B}}=10 \mathrm{~K}$ and $\epsilon_{\mathrm{dist}} / k_{\mathrm{B}}=100 \mathrm{~K}$.

\section{RESULTS}

Our simulation strategy is to utilize the models developed above to study a range of key porphyrin problems. In the first the structure of the free rings (no templates) will be analyzed while, in the second, the study will be extended to consider a number of template-bound systems. Within the latter potential coherent dynamics (so-called "caterpillar track" motion) and the effect of substitution of the metal centers will be considered. Simulations are performed at $T=300 \mathrm{~K}$ with the temperature controlled by Nosé-Hoover thermostats ${ }^{32,33}$ with an integration time-step of 0.5 fs. The simulation cell sizes reflect the experimental concentrations of around 0.0001 M.

V.A. Structural Investigations of Porphyrin Nanorings. Structural characterization of the porphyrin nanorings has been largely via a combination of NMR, X-ray crystallography, and SAXS. Of these SAXS can potentially provide the most useful information regarding the conformations adopted in the solution phase. SAXS data have been published for the free rings $\boldsymbol{c}$-P6 and $\boldsymbol{c}$-P12 $2^{8,19,20}$ and template-bound $\boldsymbol{c}$-P8.(T4) ${ }_{2}$ and $c$-P10.(T5 $)_{2}{ }^{21}$ and collected for a number of additional ring sizes. ${ }^{10}$

Our simulation strategy is to attempt to mirror the experimental procedures. To this end we first consider the structural data in reciprocal space, from which the radii of gyration may be obtained, and then turn to the extraction of real space information in the form of pair distribution functions.

1. Structure Factors. Figure 3 shows the structure factors calculated for all the investigated free porphyrin nanorings. The (Ashcroft-Langreth) structure factors are calculated directly from the Fourier components of the particle densities,

$$
S_{\alpha \beta}(k)=\left\langle A_{\alpha}^{*}(k) A_{\beta}(k)\right\rangle
$$

where

$$
A_{\alpha}(k)=\frac{1}{\sqrt{N_{\alpha}}} \sum_{i=1}^{N_{\alpha}} \exp \left(i \mathbf{k} \cdot \mathbf{r}^{i}\right)
$$

The total structure factor (observed experimentally) is given by the weighted sum of the underlying partial structure factors, 

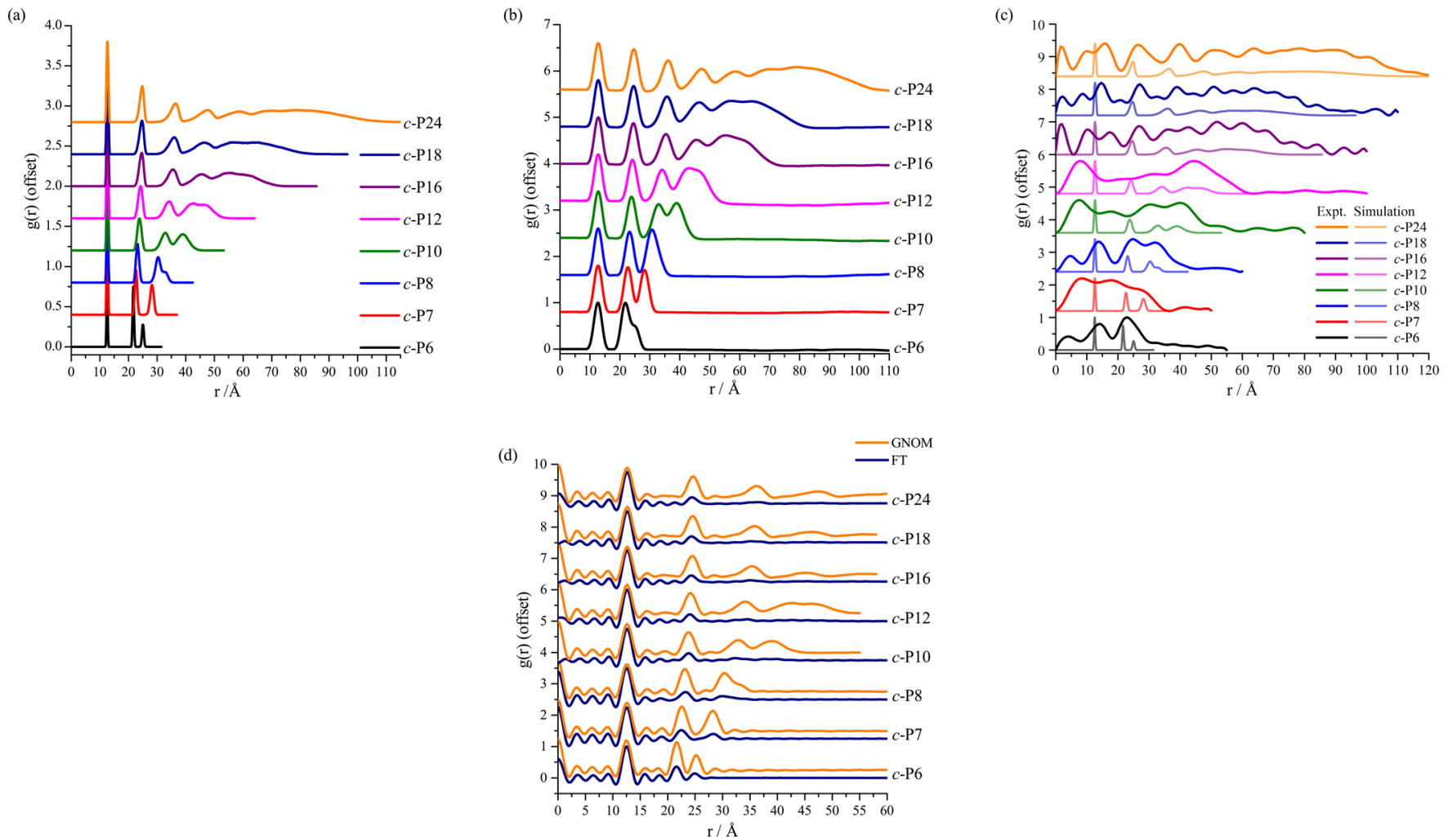

Figure 4. Evolution of the radial distribution function, $g(r)$, with nanoring size. (a) RDFs calculated from the simulation model using the metal atom positions which show peaks that become less well-defined at longer length scales as the flexibility of the rings increases. (b) RDFs calculated from Fourier transformation of the structure factors obtained from simulation. (c) RDFs obtained from the experimental SAXS data using the software package $\mathrm{GNOM}^{36}$ as described in the text. The analogous functions obtained directly from the metal atom positions are shown for clarity. (d) RDFs obtained from the simulated data using GNOM and a standard Fourier transform without a windowing function.

$$
F(k)=\sum_{\alpha} \sum_{\beta} \sqrt{c_{\alpha} c_{\beta}} f_{\alpha}(k) f_{\beta}(k)\left(S_{\alpha \beta}(k)-\delta_{\alpha \beta}\right)
$$

where $c_{\alpha(\beta)}$ and $f_{\alpha(\beta)}(k)$ are the concentration and form factors for species $\alpha(\beta)$ (taken from standard sources ${ }^{34}$ ). The X-ray patterns might reasonably be expected to be dominated by the metal atoms (and hence $F(k) \sim S_{M M}(k)$ ). Furthermore, the metal-metal structure factor will contain information regarding the basic ring conformations sampled and, since the remaining atoms form part of the same rings, then the remaining structure factors would be expected to mirror the same information. As a result, the metal-metal structure factor can be considered as a useful probe of the ring conformations. Figure 3a shows $F(k)$ for eight ring sizes obtained over $\sim 1$ ns of molecular dynamics. Figure $3 \mathrm{~b}$ shows the (normalized) experimental SAXS data for comparison. Figure 3a shows similar peak positions in the high $k$ regime $\left(k>1.0 \AA^{-1}\right.$ and highlighted in the figure) for all nanorings corresponding to relatively short distances and indicative of the similar short-range metal-metal distances present in the different rings. At lower $k$ a systematic evolution in the number and position of peaks can be seen as the nanoring size increases. This is to be expected as the number of interactions between metals and the maximum length scale will naturally increase with the number of monomers. Moreover, these would be assumed to be incremental on a geometrical basis, provided that the nanorings are rigid enough in solution.

2. Radii of Gyration. The potential model used in simulations was parametrized with experimental $R_{\mathrm{g}}$ values from free nanorings, and so excellent agreement is expected for the experimental and theoretical measurements of $R_{\mathrm{g}}$ (hence- forth $R_{\mathrm{g}}^{\mathrm{ex}}$ and $R_{\mathrm{g}}^{\mathrm{th}}$, respectively). It is nevertheless worthwhile to calculate $R_{\mathrm{g}}^{\text {th }}$ to confirm that the values calculated directly from the atom positions and via Guinier analysis from the structure factor (i.e., determined using the same method as used to analyze the experimental data) are consistent. Figure S2 shows the comparison of the two methods of calculating $R_{\mathrm{g}}^{\text {th }}$ with the results determined from the two methods in accordance. Furthermore, the values of $R_{\mathrm{g}}^{\text {th }}$ lie within the range of $R_{\mathrm{g}}^{\text {ex }}$ for all nanoring sizes, confirming that the parametrization technique was successful. Figure $S 2$ shows the values of $R_{\mathrm{g}}$ which would be expected if each ring were in a perfectly rigid circular conformation. The deviation from this rigid case is found to increase with nanoring size, indicating an increase in flexibility for the larger rings as a consequence of their higher number of degrees of freedom. This has also been observed with STM for nanorings deposited on an $\mathrm{Au}(111)$ surface. ${ }^{18}$ The upper and lower bounds of $R_{\mathrm{g}}^{\text {th }}$ from Guinier analysis are more closely spaced than the corresponding values of $R_{\mathrm{g}}^{\mathrm{ex}}$ reflecting the relative qualities of $S(k)$ in the low $k$-space regime.

3. Radial Distribution Functions. Radial distribution functions (RDFs, $g(r)$ ) are useful for quantifying structure as they contain pairwise distance information between dominant scattering entities in a system. They are also experimentally determinable, as the RDF is related to the Fourier Transform of $S(k)$. It is, however, an indirect observation of $g(r)$, accessible only through mathematical transformation of the experimental data and, as a result, is subject to potential transformation issues arising from "noise" in $S(k)$ or the necessarily finite accessible $k$ range. ${ }^{35}$ Simulation models offer an alternative (direct) method for determining $g(r)$ since the atom positions are known 
unequivocally at each time step and, as a result, these functions will naturally be free from potential transformation issues. Our strategy, therefore, is to determine the RDFs from the experimental SAXS data (requiring Fourier transformation) and from the simulation data using both the transformation (mirroring the experimental procedure) and directly from the atom positions. Since the data obtained from simulation are, in theory, more controlled than that obtained from experiment then, given the ability to generate the pair distribution functions directly from the particle positions, it should be possible to separate significant structural features from those resulting from analysis issues.

Figure 4a shows the RDFs obtained directly from the atom positions for a range of free nanorings. As the ring size increases a geometric analysis would predict a systematic increase in the number of peaks reflecting the increased range of intermetallic separations. However, while this holds true for $\boldsymbol{c}$-P6, a coalescence in the third and fourth peaks begins to be observed for $\boldsymbol{c}$-P8 and a complete amalgamation for $\boldsymbol{c}$-P10. At increasing length scales the peaks become broader and less well-defined. As the nanorings become larger, the angle between the porphyrin subunits approaches planarity and so the strain in the system is reduced which drives a greater flexibility in the nanoring and leads to an increased oscillation of the metals around their equilibrium positions. Furthermore, even if completely rigid, peaks corresponding to longer length-scales would necessarily become more closely spaced.

Figure $4 \mathrm{~b}$ shows the radial distribution functions determined from the simulated scattering data (Figure 3) via a Fourier transform using a Blackman window function to ensure $S(k)-$ 1 reaches zero at high $k$, suppressing any truncation oscillations. The data show a similar pattern of peak progressions as in Figure 4a. It is encouraging to note that both methods for calculating $g(r)$ lead to highly concordant results for the peak positions with the transformed functions showing broader peaks. Despite this, using the FT method results in the "loss" of only one major peak across all nanoring sizes, specifically the fourth peak at $33.2 \AA$ in $\mathbf{c}$-P8.

Figure $4 \mathrm{c}$ shows the radial distribution functions obtained from the SAXS data ${ }^{10}$ and processed using GNOM. ${ }^{36}$ The experimental $g(r)$ functions appear to lack the clear systematic peak progression seen in the simulated functions and which would be expected to be clear for the shorter length scale interactions. In addition, there appears to be a number of peaks which do not correspond to physically motivated intermetal separations. These peaks fall into two categories: relatively high intensity peaks at $r<10 \AA$, below any physically reasonable intramolecular metal-metal distance and periodic oscillations which percolate to high $r$. These latter oscillations appear particularly pronounced for the larger nanorings.

4. Evaluation of GNOM as a Transform Tool. Obtaining clear small-angle data which effectively probes length-scales of the order of $2-10 \mathrm{~nm}$ remains problematic. ${ }^{26,37,38}$ In Figure 4, for example, there is arguably better agreement between experiment and theory for the smaller nanorings, particularly $c$ P6 and c-P8 but all suffer from the presence of unphysical oscillations. It is, therefore, useful to determine whether these observations are a result of data quality, processing methodology, or the result of physical phenomena such as the aggregation of the nanorings in solution. Analysis software such as GNOM has been developed as traditional transform techniques fail to readily and reliably produce clear physical results from raw data. Since the scattering data obtained from simulation are highly controlled the effect of using GNOM under a range of conditions can be assessed more effectively than by using the raw experimental data.

Figure $4 \mathrm{~d}$ shows the pair distribution functions generated from the structure factors obtained from simulation transformed using both GNOM and directly without the window function. The spurious oscillations that appear in the direct FT are replicated exactly in the $g(r)$ functions generated using GNOM. There is also the appearance of a first large peak at very low $r$ as found in the experimental data. However, GNOM is clearly good at detecting and amplifying peaks at longer distances. For example, there is a return of the furthest peak in c-P8 which was "lost" on application of a windowing function.

The ramifications of these findings when considering the experimental data are less clear. In the simulated $g(r)$ it is relatively clear which peaks are generated by truncation errors due to their low intensity and periodicity. For the experimental data the separation is much less clear. An additional problem arises from the fact that both the spurious oscillations and the real peaks are highly periodic, the latter the result of the geometry of the nanorings. The ring-enforced periodicity may make detection and simple subtraction of the spurious oscillations more difficult. However, if there is an experimental $g(r)$ with obvious periodic oscillations which do not fit with any predicted model, it is fair to assume that these are likely to be artifacts of the transformation process-the best example being c-P18 in Figure 4c.

Figure S3 shows the RDFs produced by GNOM for $c$-P6 for which the SAXS data have been cut at different points at both low and high $k$ (at the respective $k$ extrema). $c$-P6 has the simplest RDF and arguably the best quality SAXS data in terms of extent in $k$-space and peak definition (see Figure $3 \mathrm{~b}$ ) and so represents the best test system. Even so, the system shows a dramatic variation in $g(r)$ as the range of the SAXS data is altered with the red line in each figure indicating the published RDF. ${ }^{19}$ Although the procedure appears to generate a relatively smooth (truncation oscillation-free) function, significant structure is lost (for example, the two peaks predicted at 21.7 and $25.1 \AA$ appear as a single feature at $r \sim 23 \AA$ ). These two peaks remain resolved if a different range of $k$ values is selected. The effective analysis of SAXS data for these systems is enormously challenging in terms of isolating the real peaks from the artifacts. Figure 5 shows the effect of varying the range of $k$ values when using GNOM to transform the structure factor generated from the simulation model. For a range less than $\Delta k$ $\sim 1.4 \AA$ the calculated distribution functions appear largely stable and the peak positions compare favorable with those obtained directly from the atom positions. For narrower $k$ ranges the peak positions become more sensitively dependent upon the precise range. Significantly, potentially, the ranges used to transform the experimental data are of the order $\Delta k \sim$ $0.6-1.3 \AA$.

V.B. Template-Bound Porphyrins. In addition to the free porphyrin nanorings, there has been increasing experimental focus on their template-bound counterparts. These may be generated either as a direct product of the synthesis in which the template acts as a scaffold for the coupling of porphyrin subunits, ${ }^{8,9,19,21}$ or via addition of template to a solution of the free nanoring. ${ }^{22}$ The considerable interest in these structures arises from the fact that the templates introduce rigidity into the otherwise flexible nanorings, leading to fully conjugated cyclic porphyrin frameworks which are shape-persistent in solution, often with enforced conformation. These molecules 


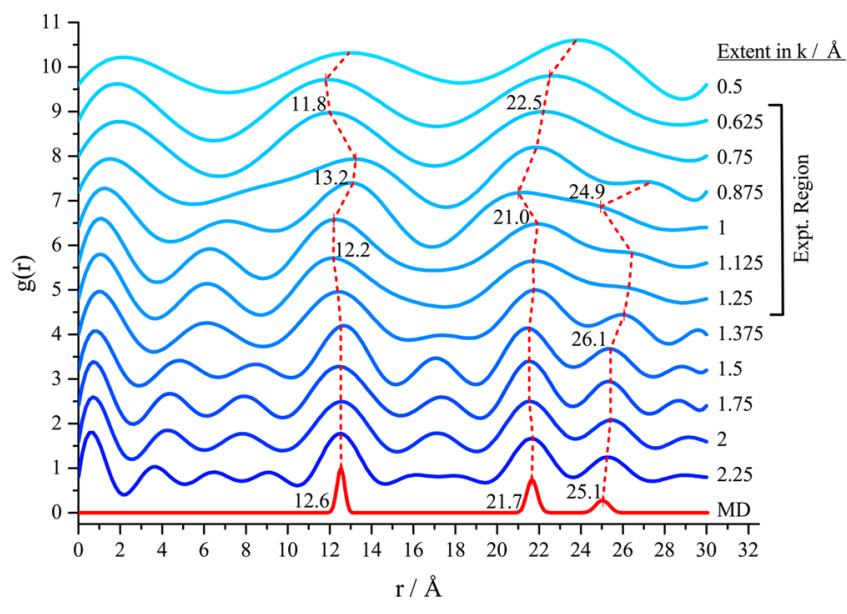

Figure 5. RDFs obtained by varying the range of $k$ values used for the simulated structure factors using GNOM for c-P6. The red dashed lines map the positions of the centers of the three expected peaks for $c$ P6 as the $k$ range for $S(k)$ is reduced. The exact peak values of selected peaks are highlighted. The peak positions are accurately determined by GNOM when $k_{\max } \geq 1.5 \AA^{-1}$. Below this value, discrepancies arise between the RDF calculated directly from positions and that from GNOM. possess potentially unique electronic and dynamical properties, which have been probed by $\mathrm{PL},{ }^{13,14} \mathrm{EPR}^{22}$ and EXSY. ${ }^{21}$ The investigation will start by examining how the introduction of a template can affect the structure of porphyrin nanorings, before trying to understand the concerted molecular motion in socalled "caterpillar track" complexes observed via EXSY. ${ }^{21}$

1. Impact of the Template on the Structure of c-P6.(T6). To understand the effect of the template we first consider the simplest of the template-bound nanoring structures, $c$-P6.(T6). The interaction between the template and the nanoring was parametrized by comparison to the interaction potential calculated from DFT between zinc or copper atoms and axially bound pyridine ${ }^{28}$ as shown in Figure S4. The optimum Morse potential parameters were found to be $D_{\mathrm{e}}=89.5 \mathrm{~kJ} \mathrm{~mol}^{-1}, r_{0}=$ $2.18 \AA$, and $\alpha=1.4 \AA^{-1}$ for zinc, and $D_{\mathrm{e}}=56.0 \mathrm{~kJ} \mathrm{~mol}^{-1}, r_{0}=$ $2.35 \AA$, and $\alpha=1.4 \AA^{-1}$ for copper. The DFT calculations generate values for the metal-pyridine interaction energies in the absence of solvent. Care must be taken in comparing the experimentally derived free energies ${ }^{27,28}$ which implcitly contain both solvent and entropic effects.

To highlight the effect of the template-nanoring interaction, simulations are performed in which the Morse potential parameter $D_{\mathrm{e}}$ is systematically altered in the range $D_{\mathrm{e}}=0-$ $200 \mathrm{~kJ} \mathrm{~mol}^{-1}$, approximately centered about the fitted value. Figure 6 shows the evolution of key structural observables as a function of $D_{\mathrm{e}}$ for $c-\mathbf{P 6}_{\mathrm{M} 6}$. As the interaction strength increases,

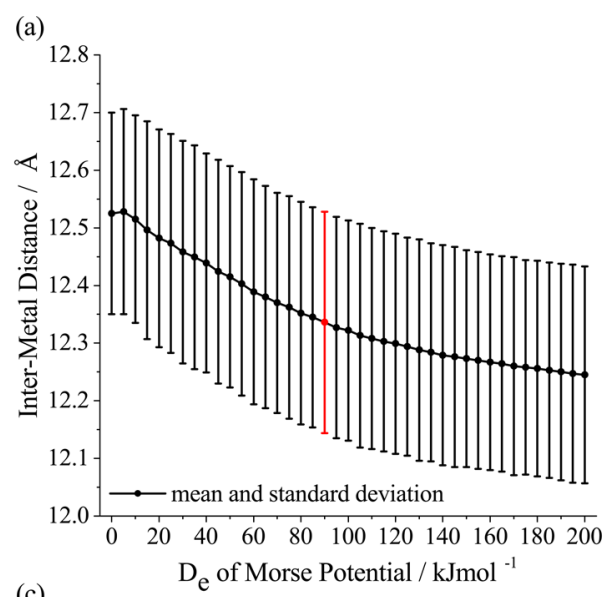

(b)
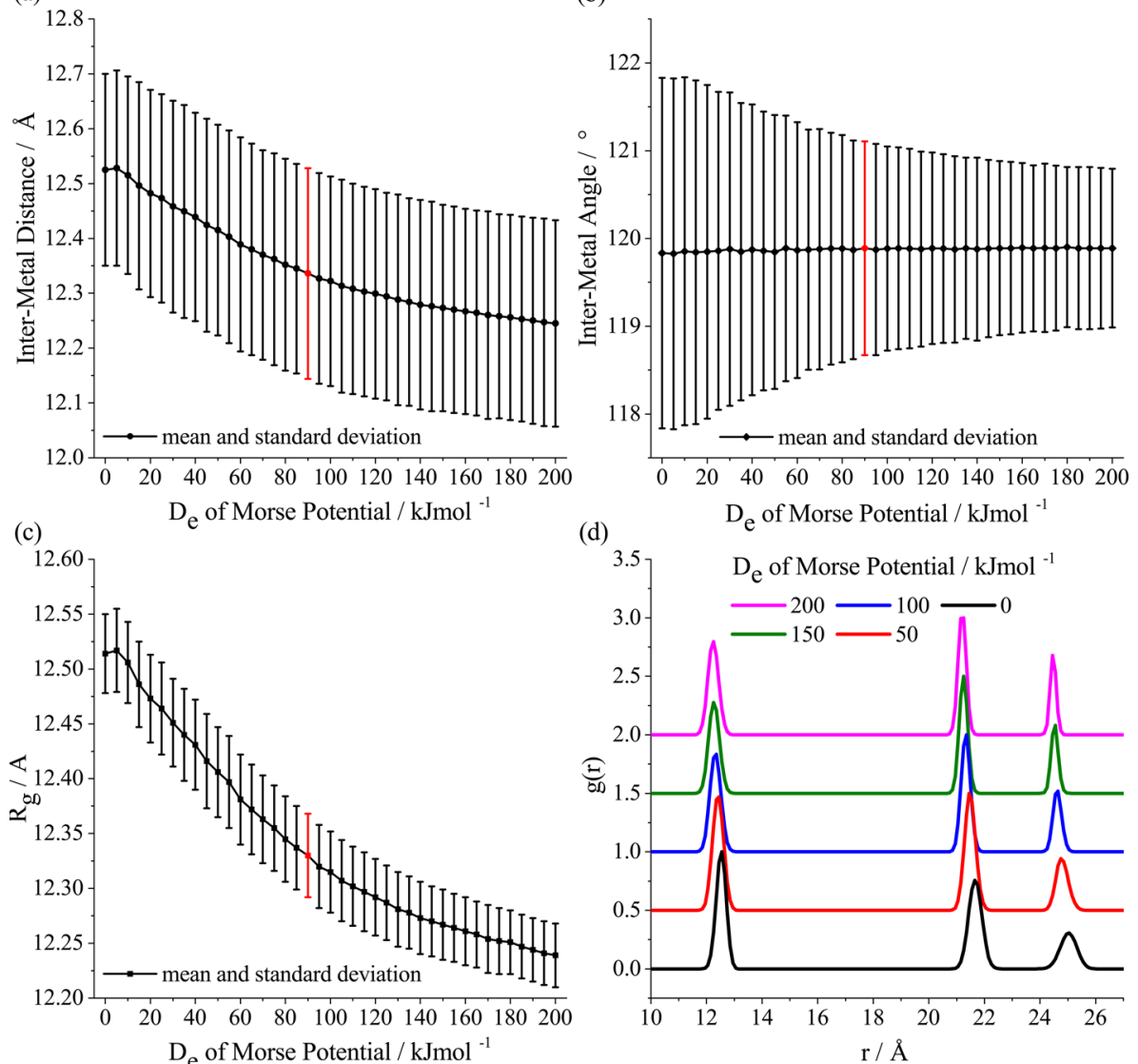

(d)

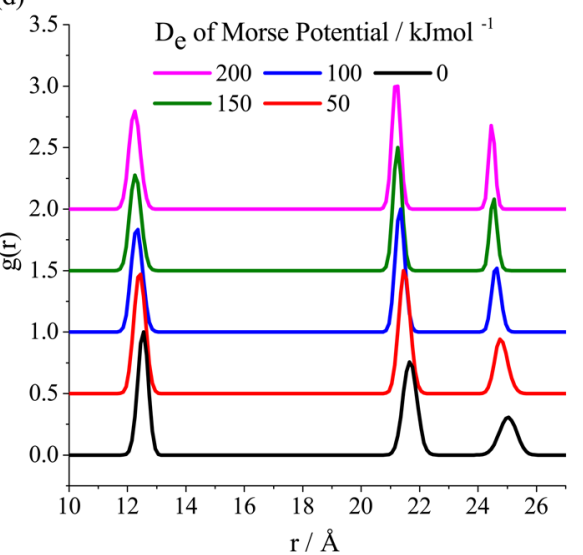

Figure 6. $(a-c)$ Variation in key structural metrics as the well depth of the Morse potential describing the metal-template interaction is varied. As the well-depth increases the metal nearest-neighbor distances decrease (a), the metal-metal-metal bond angles exhibit reduced fluctuations (b), and the radius of gyration decreases $(c)$. In panels $a-c$ the red line highlights the Morse potential well depth obtained for Zn by reference to DFT calculations. Panel d shows the respective RDFs obtained at five different metal-template well depths and highlights how the peaks narrow and display a shift to shorter length scales as $D_{\mathrm{e}}$ increases. 

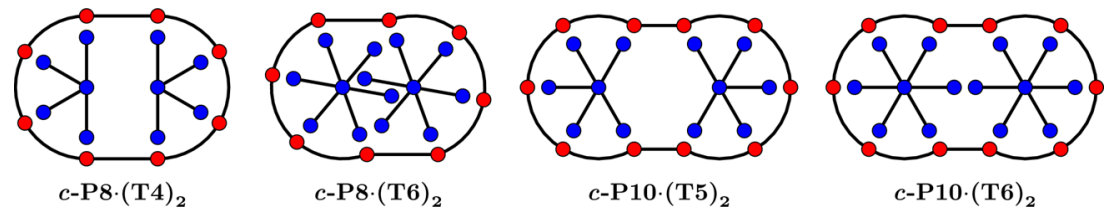

Figure 7. Schematic representations of the potential "caterpillar track" complexes: $c$-P8·(T4) $)_{2}, c$-P8·(T6 $)_{2}, c$-P10·(T5 $)_{2}$, and $c$-P10·(T6) ${ }_{2}$. The red circles highlight the location of the $\mathrm{Zn}$ centers around the nanorings.
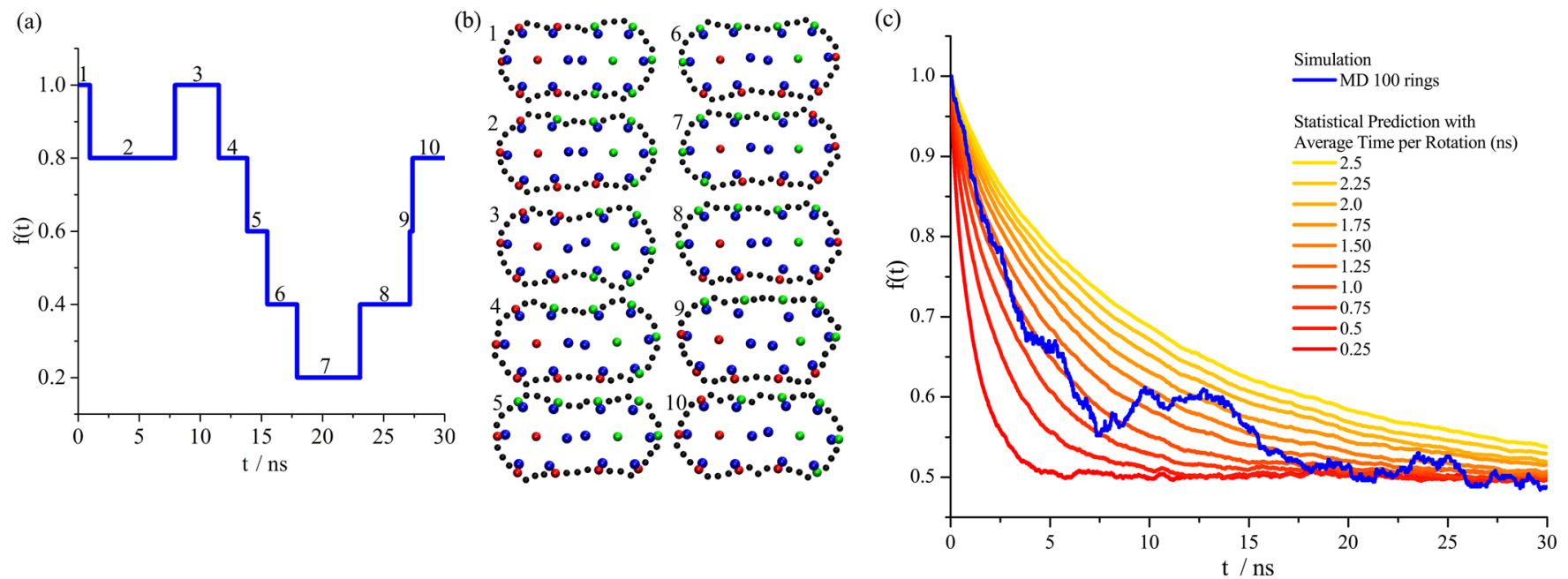

Figure 8. (a) An example time correlation function for $c$-P10.(T6) $)_{2}$ which shows moves in steps of 0.2 in both the positive and negative direction over time. (b) Molecular graphics snapshots of the conformations of each step in the TCF from panel a. Particles have been colored to show the progression in positions over time. When the color of the metal $i$ matches the color of the template it is bound to, $\delta\left[\chi_{i}(t), \chi_{i}(0)\right]=1$, else $\delta\left[\chi_{i}(t), \chi_{i}\right.$ $(0)]=0$. (c) The blue curve shows the TCF obtained from molecular dynamics simulation and averaged over configurations as described in the text. The TCF tends to 0.5 over time as any correlation with the original $(t=0)$ conformation is lost. The progressively colored yellow to red curves show that the TCG is in approximate accordance with the statistical prediction with an average rotational period between 1 and 2 ns.

the metals are pulled in toward the rigid template, facilitated by increasing distortion of the butadiyne linkages. The nearestneighbor intermetal distance is reduced (Figure 6a), accompanied by a comparative drop in the radius of gyration (Figure $6 c)$. In addition the interaction acts to tighten up the nanoring geometry such that the flexibility is reduced and the metals oscillate around the equilibrium position to a lesser extent, as demonstrated by the reduced standard deviation of the intermetal angles (Figure 6b). Both of these effects are also apparent in $g(r)$, for which there is a gradual shift in peak positions toward smaller $r$ corresponding to a reduced distance between scatterers, and a narrowing in peak width pointing to increased localization of the metal centers (Figure 6d).

2. Concerted Molecular Motion in Caterpillar Track Complexes. Recent developments have highlighted the potential for synthetic systems which display controlled motion on the molecular level (developing motors, rotors, gears, ratchets, ball-bearings, and switches). ${ }^{23}$ In addition, some template-bound porphyrin nanorings have been shown to exhibit correlated molecular motion. ${ }^{21}$ Four template-bound porphyrin systems were investigated via EXSY NMR, $c$-P8. (T4 $_{2}, c$-P8.(T6) $2, c-P 10 \cdot(T 5)_{2}$, and $c$-P10.(T6) ${ }_{2}$ (collectively referred to as "caterpillar track" (CT) complexes), and the results are shown in Figure 7. Upon proton irradiation $\mathbf{c}$-P8. (T6) $)_{2}$ showed exchange signals between environments related by $60^{\circ}$ conrotatory motion of the template molecules, while $c$ P10.(T6) $)_{2}$ showed additional signals corresponding first to $120^{\circ}$ then $180^{\circ}$ rotations, characteristic of a stepwise rotation mechanism. No exchange signals were observed on complexes without free binding sites on the template molecules (c-P8.
$\left.(\mathrm{T} 4)_{2}, c-P 10 \cdot(\mathrm{T} 5)_{2}\right)$. This implies that the CT complexes which have free template binding sites can undergo correlated motion in which the two templates move in a coherent fashion.

Molecular dynamics is potentially an excellent method with which to explore this behavior as it should be possible to visualize any coherent motion. Two systems are investigated $c$ P10.(T5 $)_{2}$ and $c$-P10.(T6) $)_{2}$, which differ only in the number of "spokes" in the respective templates. Simulations are performed on a single ring averaged over a large number of independent simulations employing different starting configurations. For each simulation each atom in the CT complex is given a different small random velocity based on a Boltzmann distribution, in order to introduce random motion into the system. To analyze any coherent motion we define two different states for the environment of a metal atom (labeled $i$ ), $\chi_{i}$, at time $t$. Each metal is either bound to template 1 or template 2 , which are arbitrarily but consistently labeled. A time correlation function (TCF) can then be constructed to monitor the relative lifetimes of the different metal environments. ${ }^{39}$ The TCF for $N_{\text {met }}$ metals is defined as follows:

$$
f(t)=\frac{1}{N_{\text {met }}} \sum_{i=1}^{N_{\text {met }}} \delta\left[\chi_{i}(t), \chi_{i}(0)\right]
$$

where $\delta$ is given by,

$$
\delta\left[\chi_{i}(t), \chi_{i}(0)\right]=\left\{\begin{array}{l}
1, \chi_{i}(t)=\chi_{i}(0) \\
0, \chi_{i}(t) \neq \chi_{i}(0) .
\end{array}\right.
$$


To facilitate the effective observation of any coherent molecular motion on a simulation time-scale the Morse potential employed to describe the metal-template interaction is weaker than that obtained by reference to the DFT calculations, using parameters of $D_{\mathrm{e}}=40 \mathrm{~kJ} \mathrm{~mol}^{-1}$ and $\alpha=1.0 \AA^{-1}$ compared with $D_{\mathrm{e}}=89.5 \mathrm{~kJ} \mathrm{~mol}^{-1}$ and $\alpha=1.4 \AA^{-1}$. Figure $8 \mathrm{a}$ shows the TCF for a single run for $\mathrm{c}$-P10.(T6) $)_{2}$, along with its corresponding conformers. At the time origin, $t_{0}$, the system is in its starting configuration (1) and $f(t)=1$. As the system conformation evolves in time, if the templates undergo a single $60^{\circ}$ conrotatory movement two metals will be transferred from their starting template to the opposing template resulting in a change in $f(t)$ of $-\frac{1+1}{10}=-0.2$, for example, moving from configuration 1 to 2 in Figure $8 \mathrm{~b}$. Similarly, subsequent rotations will lead to a change in $f(t)$ of 0.2 , the sign of which will be dependent on the direction of rotation. If the rotation transfers more metals away from their starting template associations, $f(t)$ will decrease, otherwise $f(t)$ will increase. The minimum of $f(t)$ is 0 , which corresponds to five net rotations in the same direction from the starting conformation. We can see from Figure 8a that this CT complex does indeed demonstrate stepwise rotations, the direction of which is variable.

Figure $8 \mathrm{c}$ shows the TCF obtained by averaging over 100 independent simulations. $f(t)$ decays to a limit of $f(t) \rightarrow 0.5$ expected if all possible metal-template associations are in equilibrium. This suggests that all rings display correlated motion, where the decay of $f(t)$ is illustrative of a buildup of conformations which are an increasing number of net rotations away from the starting conformation.

To further understand the rotational model a rudimentary, purely statistical model can be created to mimic the proposed behavior. The value of $f(t)$ is initialized to 1 , and a series of hypothetical rotations are made at a given average rotational period, $\tau_{\text {rot }}$ to model the effect on $f(t)$. The procedure for calculating these analytic TCFs is as follows:

1) A random number is generated in the range $0-1$. If this is greater than $\frac{1}{\tau_{\text {rot }}}$ then there is no rotation and $f(t)$ is maintained, or else a rotation is said to have occurred.

2) If a rotation has occurred then a second random number is generated to determine the direction of rotation, with 50:50 probability. Overall, therefore, $f(t)$ is changed by 0.2 with the sign of the change dependent on the chosen direction.

This procedure is repeated for 10000 random seeds, and the results averaged. Figure $8 \mathrm{~b}$ shows the results for varying $\tau_{\text {rot }}$ with comparison to the TCF obtained from MD. The TCFs generated by the two methods show some similar behavior and indicate a mean rotational period of $1-2$ ns for the present model. Comparison with the experimental frequency of $\sim 10 \mathrm{~s}^{-1}$ is difficult and merely highlights the acceleration of the dynamics promoted both by the use of high temperature and reduced template-nanoring interactions which are required to promote dynamics on the simulation time-scale.

The analogous study of $\boldsymbol{c}$-P10.(T5) $)_{2}$ shows $f(t)=1$ at all time indicating that the conformation did not deviate from its initial state on the simulation time-scale (in agreement with experiment).

Model Limitations. The correlated motion for $\mathrm{c}$-P10 $\cdot(\mathrm{T} 6)_{2}$ was observed using a reduced Morse potential interaction strength (as indicated above). Although the Morse potential has been shown to agree excellently in functional form to the potential from DFT, this is not the only consideration. The binding strength is dependent only on the distance of the template to the metal center and not their relative orientation. In real zinc porphyrins the bonding would be expected to have significant directional character which biases the nitrogen toward occupying an axial binding site. For example, the formation of five-coordinate $\mathrm{Zn}$ centers (with a single axial ligand) is relatively facile while the formation of the analogous six-coordinate (two axial ligands) species requires significant forcing conditions. ${ }^{27,40}$ The effect of this angular dependence will generally be to reduce the effective interaction strength along directions other than the preferred. As a result, it is likely that our Morse potential well depth, although appropriate to the most favorable direction of approach, overestimates the interaction energy away from this direction. For static calculations, in which the ideal angles of approach dominate, this overestimation is not a problem. However, for dynamical properties, such as the CT rotation, this may clearly be an issue.

3. Conformational Analysis of Dimetallic Porphyrin Systems. Replacing a number of zinc centers with paramagnetic copper to generate dimetallic porphyrin nanorings allows distances within the oligomers to be probed by EPR. Furthermore, the different binding affinities of the metals to the template allow the nanoring conformation to be altered with appropriate selection of the template geometry. ${ }^{22}$ The EPR focused on nanorings containing eight $\mathrm{Zn}$ and two $\mathrm{Cu}$ centers, $\boldsymbol{c}-\mathbf{P} 10_{\mathrm{Cu} 2}$, where the $\mathrm{Cu}$ species sat opposed, separated by four $\mathrm{Zn}$ units. Templates of three different geometries were inserted: $\mathbf{T 4}, \mathbf{T} \mathbf{4}_{\mathrm{X}}$, and $\mathbf{T 5}$ which allow nine possible conformations in which the $\mathrm{Cu}$ and $\mathrm{Zn}$ atoms show differing connectivities to the respective templates (Figure 9). The three

1)
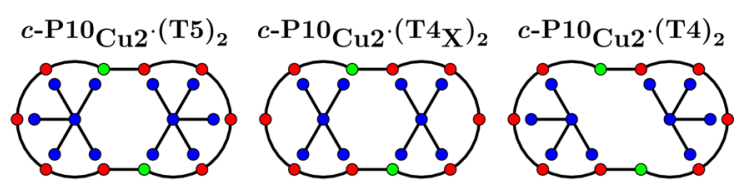

2)
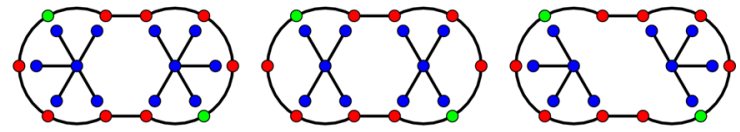

3)
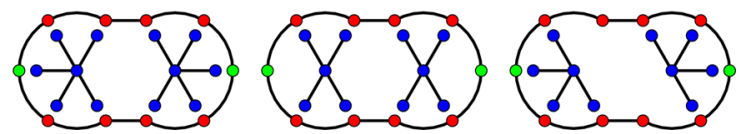

Figure 9. Schematic representation of the possible conformers for the template-bound $\boldsymbol{c}-\mathrm{P} 10_{\mathrm{Cu} 2}$ complexes. Metals colored green indicate $\mathrm{Cu}$ positions while those colored red mark the $\mathrm{Zn}$ locations.

samples were probed using double electron-electron resonance (DEER) spectroscopy which measures the dipolar coupling between the unpaired electrons on the $\mathrm{Cu}$ centers and hence their separation.

Our simulation strategy is to model a generic ring system in which the interaction strength of the metal centers is systematically varied.

Molecular dynamics simulations were performed for each of the nine starting conformers shown in Figure 9. The Zntemplate Morse potential well depth was fixed at $D_{\mathrm{e}}=89.5 \mathrm{~kJ}$ $\mathrm{mol}^{-1}$ (as obtained by fit to the DFT results from section VB1). The analogous fit to the $\mathrm{Cu}$-centered DFT calculations gives a best fit of $D_{\mathrm{e}}=56 \mathrm{~kJ} \mathrm{~mol}^{-122}$ although the free energy of 

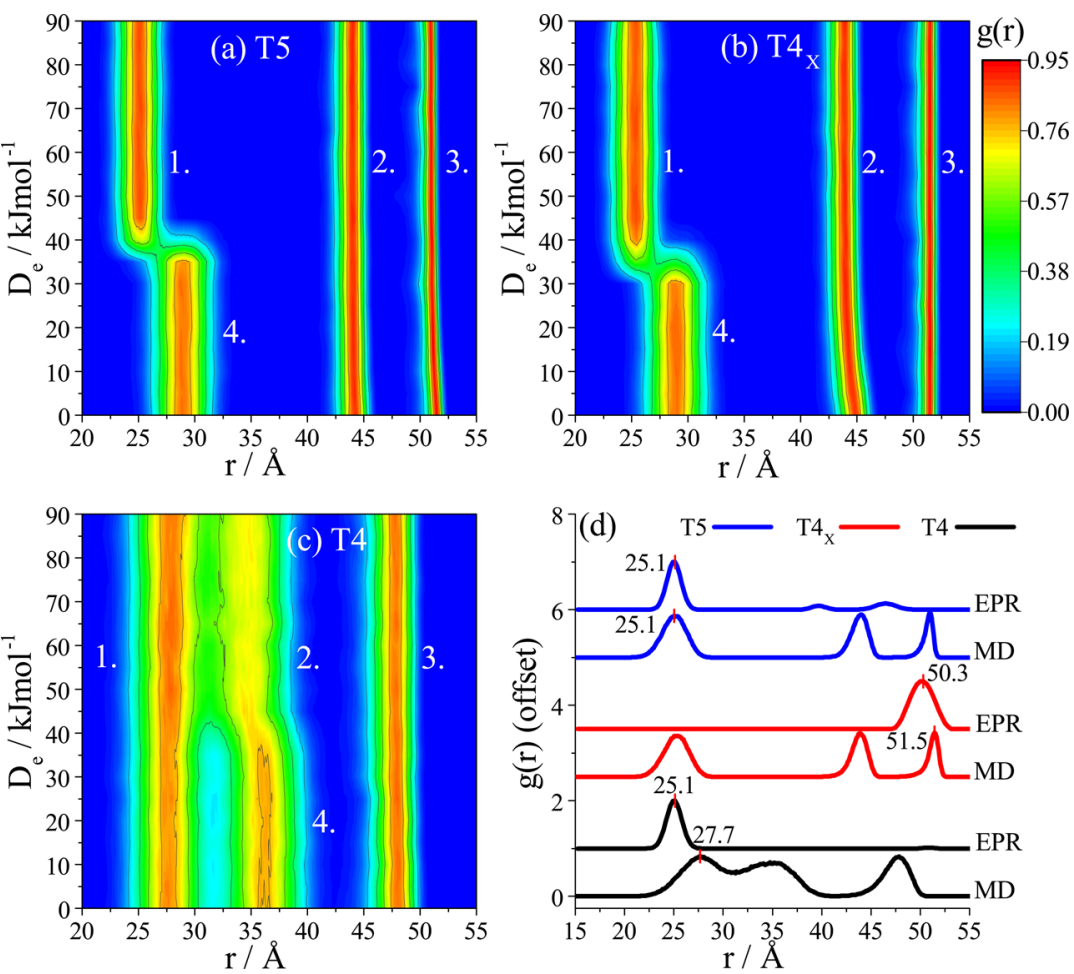

Figure 10. $(\mathrm{a}-\mathrm{c})$ Radial distribution functions, $g(r)$, for $\mathrm{c}-\mathrm{P} 10_{\mathrm{M} 2}$ with different templates (as indicated) as the Cu-template dissociation energy, $D_{\mathrm{e}}$, is varied as the $\mathrm{Zn}$-template energy is kept constant. (d) Compares the distance distribution of $\boldsymbol{c}-\mathbf{P} 10_{\mathrm{Cu} 2}$ with each template measured by EPR ${ }^{22}$ and simulated via MD. Specific peak positions are highlighted.

formation for the $\mathrm{Cu}$-pyridine system has been estimated as $\sim 6.2 \mathrm{~kJ} \mathrm{~mol}^{-1}$ from a consideration of differences in formationfree energies. ${ }^{28}$ To rationalize the effect of two different metal binding interaction strengths the Cu-template term is systematically varied from. $D_{\mathrm{e}}=0-90 \mathrm{~kJ} \mathrm{~mol}^{-1}$, again encompassing the ideal fitted value. The results for each of the templates have been combined and can be found in Figure 10(a)-(c). At each value of $D_{e}$ simulations are performed started from each of the three conformers with the resulting distribution functions averaged over these runs. T5 and $\mathbf{T} \mathbf{4}_{\mathbf{X}}$ show very similar progressions in $g(r)$ as $D_{\mathrm{e}}$ is varied. At high $D_{\mathrm{e}}\left(D_{\mathrm{e}}>45 \mathrm{~kJ}\right.$ $\mathrm{mol}^{-1}$ ) the conformers are analogous to those in Figure 9. However, below this value a conformational change is initiated. First there is a simultaneous depletion in the peak for conformer 1 and a corresponding buildup of a new peak, until at $D_{\mathrm{e}} \sim 35 \mathrm{~kJ} \mathrm{~mol}^{-1}$ there is complete replacement. This is due to detachment of a template leg from the metal and the resulting rotation of the template around to relieve strain in the backbone (see Figure 11a). There is much less of an effect for conformers 2 and 3. Even if there is a low binding potential, the metal is still held in close proximity to the template by virtue of the geometry of the system. Hence only small deviations at very low values of $D_{\mathrm{e}}$ are seen. The exception is for conformer 3 of $\mathbf{T} 4_{\mathbf{X}}$. As this has no binding to the metal in question, we would expect no variation in $g(r)$, as observed.

The results for T4 differ markedly to the other two systems. Peaks appear much broader, pointing to increased flexibility in the system, and the positions for all three conformers are shifted. These shifts are attributed to adoption of rotated conformers of a similar form to the low $D_{\mathrm{e}}$ species seen for conformer 1 of $\mathbf{T 5}$ and $\mathbf{T} \mathbf{4}_{\mathrm{X}}$, as in Figure $11 \mathrm{~b}$. It is now conformer 2 which displays the large change at low $D_{\mathrm{e}}$, and there is a particularly broad peak owing to the fact that two (a)
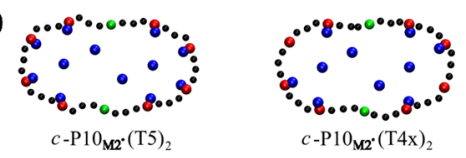

(b)

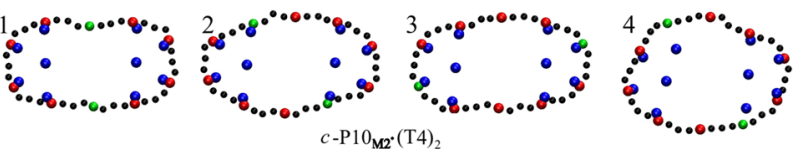

Figure 11. (a) Molecular graphics snapshots of conformer 4 of $c$ $\mathbf{P 1 O}_{\mathrm{M} 2} \cdot(\mathrm{T} 5)_{2}$ (left) and $c-\mathrm{P1O}_{\mathrm{M} 2} \cdot\left(\mathrm{T} \mathbf{4}_{\mathrm{X}}\right)_{2}$ (right). With lower $D_{\mathrm{e}}$ the template legs may uncouple from the metal, facilitating rotation in order to relieve strain. (b) Molecular graphics snapshots of the conformers of $c-\mathrm{PlO}_{\mathrm{M} 2} \cdot(\mathrm{T} 4)_{2}$, which are rotated relative to the starting conformations in Figure 9.

consecutive porphyrin units are unbound to the template. As an aside, it is noted with interest that the region in which we begin to detect dissociation of the metal from the template in all cases is the same potential which led to the caterpillar track motion discussed earlier.

The results from MD can now be compared to those from with EPR for the specific case of $\boldsymbol{c}$-P10 $\mathbf{C u 2}$ (Figure 10, panel d). There are however two important things to note before doing so. First $\mathrm{MD}$ simulation does not capture the relative abundance of each conformer in solution as the simulations are performed on the individual conformers rather than, for example, starting from an ensemble of a single conformer and establishing a full conformational equilibrium. In contrast the EPR data will only contain distances pertaining to the conformers favored by equilibrium, that is, those which maximize template- $\mathrm{Zn}$ binding; the simulation will have an equal contribution from each. Second, when EPR is used, only 
shorter distances can generally be determined with high precision. The errors for longer distances are larger due to the limited length of the DEER trace. ${ }^{22}$ This being the case, very good agreement is seen for the shortest length scales for both $c-P 10_{\mathrm{Cu} 2} \cdot(\mathrm{T} 5)_{2}$ and $c-\mathrm{P} 10_{\mathrm{Cu} 2} \cdot\left(\mathrm{T} 4_{\mathrm{X}}\right)_{2}$. However, there is a discrepancy for $c-P 10_{\mathrm{Cu} 2} \cdot(\mathrm{T} 4)_{2}$. The DEER analysis shows a much more well-defined peak 2.6 Å lower than predicted from the MD simulations. The exact reason for this difference has not yet been isolated, but it is likely that the potential model is not capturing the flexibility accurately for these niche cases where most metals occupy highly strained positions but a few remain free. These free metals then sit slightly further out than expected in order to alleviate the angle strain imposed by the model.

4. Angular Variation in Dimetallic Porphyrin Systems. The interpretation of photoluminescence spectroscopy measurements on a range of template-bound nanorings resulted in a proposed reduction in the longer intermetallic length-scales arising from relatively facile bending and twisting mode. ${ }^{14}$ However, EPR measurements appear consistent with the retention of largely planar configurations. ${ }^{22}$ Figure 12 a shows

(a)

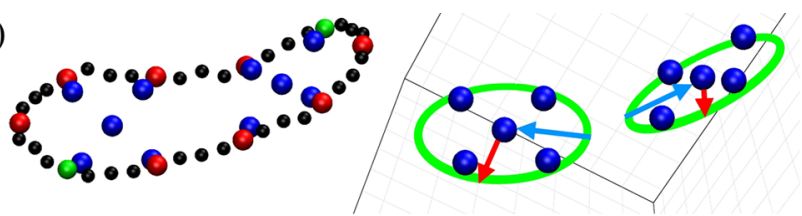

(b)

(c)
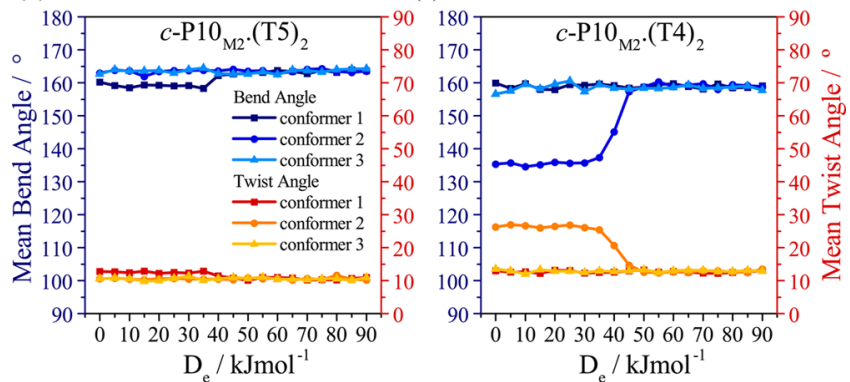

Figure 12. (a) (Left) molecular graphics snapshot of the system $c$ $\mathrm{P10}_{\mathrm{Cu} 2} \cdot(\mathrm{T} 5)_{2}$; (right) the templates are superimposed on the best-fit planes with the vectors used in the subsequent calculation of the bending angles shown in blue, while those for the twisting angle are displayed in red. (b, c) evolution of the mean bond (blue lines) and twist (red lines) in the T5 and T4 template-bound complexes respectively as the metal-template interaction strength is increased.

the basis for the calculation used to investigate these deformations in the present work. For each nanoring we define two planes which contain the templates. To calculate the bending angle, the dot product is taken of two vectors which originate at the closest distance of approach between the planes and terminate at the centers of the templates (blue arrows). To calculate the twisting angle, the dot product is used of the two vectors contained in the planes orthonormal to those previous (red arrows). For a planar complex we would therefore measure bending and twisting angles of $180^{\circ}$ and $0^{\circ}$, respectively.

Figure 12 panels $\mathrm{b}$ and $\mathrm{c}$ show the respective bending and twisting angles for $c-\mathrm{P} 10_{\mathrm{M} 2} \cdot(\mathrm{T} 5)_{2}$ and $c-\mathrm{P}_{10} \mathrm{M} \cdot(\mathrm{T} 4)_{2}$ again as a function of $D_{\mathrm{e}}$. The $c-\mathrm{P} 10_{\mathrm{M} 2} \cdot\left(\mathbf{T} \mathbf{4}_{\mathrm{X}}\right)_{2}$ complex shows a similar pattern to that for $c-\mathrm{P} 10_{\mathrm{M} 2} \cdot(\mathrm{T} 5)_{2}$ and is omitted here. Focusing initially on the specific parameters associated with $\mathrm{Cu}, D_{\mathrm{e}}=56$ $\mathrm{kJ} \mathrm{mol}^{-1}$, only small deviations from planarity are seen, with twist and bend angles of $11^{\circ} / 164^{\circ}$, respectively for the T5 complex and $13^{\circ} / 159^{\circ}$ for T4. The extra flexibility of the T4 complex is expected considering the lack of template binding to the metals at the center of the nanoring. There has been one previous attempt to calculate these angles for $c$-P10.(T5 $)_{2}$ with a molecular mechanics approach which led to twist and bend angles of $68^{\circ} / 126^{\circ 14}$, respectively. However, these angles would appear to be somewhat dramatic in the contest of the EPR results.

Below the "dissociation point" (effectively the energy at which the template "leg" decouples), a small loss in rigidity is seen for conformer 1 of $c-\mathbf{P 1 0}_{\mathrm{M}_{2}} \cdot(\mathrm{T} 5)_{2}$, and the angular values closely resemble those of $c-\mathrm{P10}_{\mathrm{Cu} 2} \cdot(\mathrm{T} 4)_{2}$, highlighting the similar structure of the conformers (Figure 11a,b). There is a much larger shift for $\mathrm{c}-\mathrm{P} 1 \mathbf{1 0}_{\mathrm{M} 2} \cdot(\mathrm{T} 4)_{2}$, which displays values of $27^{\circ} / 135^{\circ}$ for the twist and bend angles, again reflective of the fact that two consecutive porphyrin units become unbound to the template. Finally, as would be expected, this "dissociation point" is of the order of the $D_{\mathrm{e}}$ used earlier to promote CT-like motion on the simulation time-scale.

\section{CONCLUSIONS}

Coarse-grained models have been developed to systematically study a range of both template-bound and free porphyrin nanorings. The use of relatively simple energy functions to describe the basic interactions allows a range of systems to be studied in a physically transparent manner. Moreover, the relative model simplicity allows direct observation of complex dynamical processes such as the so-called "caterpillar track" rotational motion, allowing the key structural and interaction origins to be isolated and analyzed. Key interaction potential terms have been varied in a systematic manner which allows the relative stability of different potential conformers to be assessed and rationalized.

\section{ASSOCIATED CONTENT}

\section{Supporting Information}

The Supporting Information contains . The Supporting Information is available free of charge on the ACS Publications website at DOI: 10.1021/acs.jpca.7b05279.

Details on model parametrization, simulation details, experimental details, processing of SAXS data, additional information on the radii of gyration, use of GNOM, and the DFT calculations (PDF)

\section{AUTHOR INFORMATION}

\section{Corresponding Author}

*E-mail: mark.wilson@chem.ox.ac.uk.

ORCID

Harry L. Anderson: 0000-0002-1801-8132

Mark Wilson: 0000-0003-4599-7943

Notes

The authors declare no competing financial interest.

\section{ACKNOWLEDGMENTS}

The authors are grateful to Professor Christiane R. Timmel and Dr. Sabine Richert for useful discussions and for supplying experimental DEER data and computational DFT data. We thank the following organizations for support: the Engineering and Physical Sciences Research Council (EPSRC), the European Research Council (Grant 320969), the Clarendon 
Fund of the University of Oxford (D.V.K.) and Royal Thai Government Scholarship (N.K.). We thank Diamond Light Source for an award of beamtime on I22. We are grateful for support from the EPSRC Centre for Doctoral training, Theory and Modelling in Chemical Sciences, under Grant EP/ L015722/1. This paper conforms to the RCUK data management requirements.

\section{REFERENCES}

(1) Kuester, W. Beitraege zur Kenntnis des Bilirubins und Haemins. Hoppe-Seyler's Z. Physiol. Chem. 1912, 82, 463.

(2) Sheldon, R. A. Metalloporphyrins in Catalytic Oxidations; Marcel Dekker, 1994.

(3) McDermott, G.; Prince, S. M.; Freer, A. A.; HawthornthwaiteLawless, A. M.; Papiz, M. Z.; Cogdell, R. J.; Isaacs, N. W. Crystal structure of an integral membrane light-harvesting complex from photosynthetic bacteria. Nature 1995, 374, 517-521.

(4) Aratani, N.; Kim, D.; Osuka, A. Discrete cyclic porphyrin arrays as artificial light-harvesting antenna. Acc. Chem. Res. 2009, 42, 19221934.

(5) Li, J.; Ambroise, A.; Yang, S. I.; Diers, J. R.; Seth, J.; Wack, C. R.; Bocian, D. F.; Holten, D.; Lindsey, J. S. Template-directed synthesis, excited-state photodynamics, and electronic communication in a hexameric wheel of porphyrins. J. Am. Chem. Soc. 1999, 121, 89278940.

(6) Hoffmann, M.; Wilson, C. J.; Odell, B.; Anderson, H. L. Template-directed synthesis of a $\pi$;-conjugated porphyrin nanoring. Angew. Chem., Int. Ed. 2007, 46, 3122-3125.

(7) Hoffmann, M.; Kärnbratt, J.; Chang, M. H.; Herz, L. M.; Albinsson, B.; Anderson, H. L. Enhanced $\pi$ conjugation around a porphyrin[6] nanoring. Angew. Chem., Int. Ed. 2008, 47, 4993-4996.

(8) O'Sullivan, M. C.; et al. Vernier templating and synthesis of a 12porphyrin nano-ring. Nature 2011, 469, 72-75.

(9) Kondratuk, D. V.; Perdigao, L. M. A.; O’Sullivan, M. C.; Svatek, S.; Smith, G.; O’Shea, J. N.; Beton, P. H.; Anderson, H. L. Two vernier-templated routes to a 24-porphyrin nanoring. Angew. Chem., Int. Ed. 2012, 51, 6696-6699.

(10) Malfois, M.; Kamonsutthipaijit, N.; Kondratuk, D.; Anderson, H. L. I22 Beamline Reports: Raw SAXS Data; Diamond, 2014.

(11) Kondratuk, D. V.; Perdigão, L. M. A.; Esmail, A. M. S.; O’Shea, J. N.; Beton, P. H.; Anderson, H. L. Supramolecular nesting of cyclic polymers. Nat. Chem. 2015, 7, 317-322.

(12) Parkinson, P.; Knappke, C. E. I.; Kamonsutthipaijit, N.; Sirithip, K.; Matichak, J. D.; Anderson, H. L.; Herz, L. M. Ultrafast energy transfer in biomimetic multistrand nanorings. J. Am. Chem. Soc. 2014, 136, 8217-8220.

(13) Yong, C.-K.; et al. Ultrafast delocalization of excitation in synthetic light-harvesting nanorings. Chem. Sci. 2015, 6, 181-189.

(14) Gong, J. Q.; Parkinson, P.; Kondratuk, D. V.; Gil-Ramírez, G.; Anderson, H. L.; Herz, L. M. Structure-directed exciton dynamics in templated molecular nanorings. J. Phys. Chem. C 2015, 119, 64146420.

(15) Kim, Y.; et al. A strong regioregularity effect in self-organizing conjugated polymer films and high-efficiency polythiophene:fullerene solar cells. Nat. Mater. 2006, 5, 197-203.

(16) Park, S. H.; Roy, A.; Beaupre, S.; Cho, S.; Coates, N.; Moon, J. S.; Moses, D.; Leclerc, M.; Lee, K.; Heeger, A. J. Bulk heterojunction solar cells with internal quantum efficiency approaching $100 \%$. Nat. Photonics 2009, 3, 297-303.

(17) Svatek, S. A.; Perdigao, L. M. A.; Stannard, A.; Wieland, M. B.; Kondratuk, D. V.; Anderson, H. L.; O'Shea, J. N.; Beton, P. H. Mechanical stiffening of porphyrin nanorings through supramolecular columnar stacking. Nano Lett. 2013, 13, 3391-3395.

(18) Wieland, M. B.; Perdigão, L. M. A.; Kondratuk, D. V.; O’Shea, J. N.; Anderson, H. L.; Beton, P. H. Height dependent molecular trapping in stacked cyclic porphyrin nanorings. Chem. Commun. 2014, 50, 7332-5.
(19) Sprafke, J. K.; et al. Belt-shaped $\pi$-systems: Relating geometry to electronic structure in a six-porphyrin nanoring. J. Am. Chem. Soc. 2011, 133, 17262-17273.

(20) Kondratuk, D. V.; Sprafke, J. K.; O’Sullivan, M. C.; Perdigao, L. M. A.; Saywell, A.; Malfois, M.; O'Shea, J. N.; Beton, P. H.; Thompson, A. L.; Anderson, H. L. Vernier-templated synthesis, crystal structure, and supramolecular chemistry of a 12-porphyrin nanoring. Chem. - Eur. J. 2014, 20, 12826-12834.

(21) Liu, S.; Kondratuk, D. V.; Rousseaux, S. A. L.; Gil-Ramírez, G.; O'Sullivan, M. C.; Cremers, J.; Claridge, T. D. W.; Anderson, H. L. Caterpillar track complexes in template-directed synthesis and correlated molecular motion. Angew. Chem., Int. Ed. 2015, 54, $5355-5359$.

(22) Richert, S.; Cremers, J.; Anderson, H. L.; Timmel, C. R. Exploring Template-Bound Dinuclear Copper Porphyrin Nanorings by EPR Spectroscopy. Chem. Sci. 2016, 7, 6952-6960.

(23) Kay, E. R.; Leigh, D. A.; Zerbetto, F. Synthetic molecular motors and mechanical machines. Angew. Chem., Int. Ed. 2007, 46, 72-191.

(24) Rousseaux, S. A. L.; Gong, J. Q.; Haver, R.; Odell, B.; Claridge, T. D. W.; Herz, L. M.; Anderson, H. L. Self-Assembly of Russian Doll Concentric Porphyrin Nanorings. J. Am. Chem. Soc. 2015, 137, 12713-12718.

(25) Jiang, H.-W.; Tanaka, T.; Mori, H.; Park, K. H.; Kim, D.; Osuka, A. Cyclic 2,12-Porphyrinylene Nanorings as a Porphyrin Analogue of Cycloparaphenylenes. J. Am. Chem. Soc. 2015, 137, 2219.

(26) Lovett, J. E.; Hoffmann, M.; Cnossen, A.; Shutter, A. T. J.; Hogben, H. J.; Warren, J. E.; Pascu, S. I.; Kay, C. W. M.; Timmel, C. R; Anderson, H. L. Probing flexibility in porphyrin-based molecular wires using double electron electron resonance. J. Am. Chem. Soc. 2009, 131, 13852-13859.

(27) Favereau, L.; Cnossen, A.; Kelber, J. B.; Gong, J. Q.; Oetterli, R. M.; Cremers, J.; Herz, L. M.; Anderson, H. L. Six-Coordinate Zinc Porphyrins for Template-Directed Synthesis of Spiro-Fused Nanorings. J. Am. Chem. Soc. 2015, 137, 14256-14259.

(28) Cremers, J.; Richert, S.; Kondratuk, D.; Claridge, T.; Timmel, C. R; Anderson, H. L. Nanorings with copper(II) and zinc(II) centers: forcing copper porphyrins to bind axial ligands in heterometallated oligomers. Chem. Sci. 2016, 7, 6961-6968.

(29) Morse, P. Diatomic Molecules According to the Wave Mechanics. II. Vibrational Levels. Phys. Rev. 1929, 34, 57-64.

(30) Feigin, L. A.; Svergun, D. I. Structure Analysis by Small-Angle Xray and Neutron Scattering; Plenum Press, 1987.

(31) Kikhney, A. G.; Svergun, D. I. A practical guide to small angle Xray scattering (SAXS) of flexible and intrinsically disordered proteins. FEBS Lett. 2015, 589, 2570.

(32) Nosé, S. A molecular dynamics method for simulations in the canonical ensemble. Mol. Phys. 1984, 52, 255-268.

(33) Hoover, W. G. Canonical dynamics: Equilibrium phase-space distributions. Phys. Rev. A: At., Mol., Opt. Phys. 1985, 31, 1695-1697.

(34) Cromer, D. T.; Waber, J. T. International Tables for X-ray Crystallography; Kynoch Press: Birmingham, 1974.

(35) Fischer, H. E.; Barnes, A. C.; Salmon, P. S. Neutron and x-ray diffraction studies of liquids and glasses. Rep. Prog. Phys. 2006, 69, 233.

(36) Svergun, D. I. Determination of the regularization parameter in indirect-transform methods using perceptual criteria. J. Appl. Crystallogr. 1992, 25, 495-503.

(37) Lei, S.; Ver Heyen, A.; De Feyter, S.; Surin, M.; Lazzaroni, R.; Rosenfeldt, S.; Ballauff, M.; Lindner, P.; Moessinger, D.; Hoeger, S. Two-Dimensional Oligo(phenylene-ethynylene-butadiynylene)s: AllCovalent Nanoscale Spoked Wheels. Chem. - Eur. J. 2009, 15, 2518.

(38) Easwaramoorthi, S.; et al. Structure-property relationship for two-photon absorbing multiporphyrins: Supramolecular assembly of highly-conjugated multiporphyrinic ladders and prisms. J. Phys. Chem. A 2008, 112, 6563-6570.

(39) Wilson, M. The effects of topology on the structural, dynamic and mechanical properties of network-forming materials. J. Phys.: Condens. Matter 2012, 24, 284114.

(40) Abraham, R.; Leighton, P.; Sanders, J. K. M. Coordination chemistry and geometries of some 4, 4'-bipyridyl-capped porphyrins. 
Proton-and ligand-induced switching of conformations. J. Am. Chem.

Soc. 1985, 107, 3472-3478. 